\title{
Application of the 0-1 Test for Chaos to Experimental Data
}

\author{
Ian Falconer * Georg A. Gottwald ${ }^{\dagger} \quad$ Ian Melbourne ${ }^{\ddagger}$ \\ Kjetil Wormnes ${ }^{\S}$
}

17 October 2006; Revised 14 March 2007

\begin{abstract}
A reliable and efficient method to distinguish between chaotic and nonchaotic behaviour in noise-contaminated, but essentially stationary and deterministic, time series data has far reaching applications. Recently, we proposed a new method for detecting chaos which applies directly to the time series data and does not require phase space reconstruction. To illustrate the effectiveness of the method for experimental data, we analyse data from a bipolar motor.
\end{abstract}

The standard technique to distinguish between regular and chaotic dynamics in deterministic time series data is to calculate the maximal Lyapunov exponent $[1,7$, 14]. In the case of physical and biological applications, where the underlying dynamics is unknown, phase space reconstruction $[1,7,16,19,18]$ is a necessary first step to compute Lyapunov exponents. However, there are problems inherent in phase space reconstruction as discussed in detail in $[6,17]$.

Recently, we proposed a new method [9] to detect chaos which applies directly to the time series data and does not require phase space reconstruction. Moreover, the dimension and origin of the dynamical system are irrelevant. The input is the time series data and the output is zero or one depending on whether the dynamics is nonchaotic or chaotic. This zero-one test for chaos is equally applicable to maps, ordinary and partial differential equations, and generally to data sets arising from deterministic systems. Of utmost importance is the effectiveness of the method for experimental

\footnotetext{
*School of Physics, University of Sydney, NSW 2006, Australia

${ }^{\dagger}$ Mathematics and Statistics, University of Sydney, NSW 2006, Australia

${ }^{\ddagger}$ Mathematics and Statistics, University of Surrey, Guildford, Surrey GU2 7XH, UK

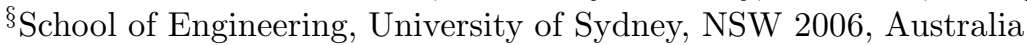


data. To illustrate our method we analyze data coming from an experimental set up of a bipolar motor in an alternating magnetic field.

We first describe our test. Consider a scalar observable $\Phi(n)$. In an experiment, $\Phi(n)$ is a discrete set of measurement data. Choose $c>0$ and define

$$
p(n)=\sum_{j=1}^{n} \Phi(j) \cos j c
$$

for $n=1,2, \ldots$ We claim that (i) $p(n)$ is bounded if the underlying dynamics is non-chaotic (e.g. periodic or quasiperiodic) and (ii) $p(n)$ behaves asymptotically like Brownian motion if the underlying dynamics is chaotic. Later on, we briefly explain the justification behind these claims. For the moment, we suppose that the claims are correct and show how to proceed.

The form of our test (1) which involves only the observable $\Phi(n)$ highlights the universality of the test; the origin and nature of the data fed into the diagnostic system (1) is irrelevant for the test. The method is independent of the observable; almost any choice of $\Phi$ will suffice [9]. Moreover, the dimension of the underlying dynamical system does not pose practical limitations on the method as is the case for traditional methods involving phase-space reconstruction [17]. Of course, it is necessary (as with all other methods) that (i) initial transients have died out so that the trajectories are on (or close to) the attractor at time zero, and (ii) the time series is long enough to allow for asymptotic behaviour of $p(n)$. In particular, it is required that the data is essentially stationary as well as deterministic. High-dimensionality of the attractor may impact on (ii), since the time series must be sufficient in length that the dynamics explores enough of the attractor; but this is an intrinsic part of the problem and not a drawback of the method.

To determine the character of the growth of the function $p(n)$ defined in (1), i.e. its diffusive behaviour, it is natural to look at the mean square displacement of $p(n)$, defined to be $M(n)=\lim _{N \rightarrow \infty} \frac{1}{N} \sum_{j=1}^{N}[p(j+n)-p(j)]^{2}$. If the behaviour of $p(n)$ is asymptotically Brownian, i.e. the underlying dynamics is chaotic, then $M(n)$ grows linearly in time; if the behaviour is bounded, i.e. the underlying dynamics is nonchaotic, then also $M(n)$ is bounded. The asymptotic growth rate $K$ of $M(n)$ can be numerically determined by means of linear regression of $\log M(n)$ versus $\log n$. This allows for a clear distinction of a non-chaotic and a chaotic system as either $K=0$ or $K=1$.

To justify the $0-1$ test for chaos, note that the function $p(n)$ can be viewed as a 
component of the solution to the skew product system

$$
\begin{aligned}
& \theta(n+1)=\theta(n)+c, \\
& p(n+1)=p(n)+\Phi(n) \cos \theta(n), \\
& q(n+1)=q(n)+\Phi(n) \sin \theta(n) .
\end{aligned}
$$

Here $(\theta, p, q)$ represent coordinates on the Euclidean group $E(2)$ of rotations $\theta$ and translations $(p, q)$ in the plane. It has been shown [13] that typically the dynamics on the group extension is sub-linear and is (i) bounded if the underlying dynamics is non-chaotic, (ii) unbounded (but sub-linear) if the underlying dynamics is chaotic. Moreover, the $p$ and $q$ components each behave asymptotically like Brownian motion on the line if the chaotic attractor is uniformly hyperbolic [8]. A non-degeneracy result [13] ensures that for chaotic dynamics the variance of the Brownian motion is nonzero for almost all choices of $c>0$ and observable $\Phi$. Recent work $[12,5]$ indicates that these statements remain valid for large classes of non-uniformly hyperbolic systems, such as Hénon-like attractors. Roughly speaking, to obtain $K=1$ it suffices that the underlying system has a faster than quadratic rate of decay of correlations $[2,4,9]$.

One might ask why it is not better to work, instead of the $E(2)$-extension, with the simpler $\mathbb{R}$-extension $p(n+1)=p(n)+\Phi(n)$, which can again be used to detect for chaos [9]. However, $p(n)$ grows linearly and it is necessary to subtract off the linear term before computing $M(n)$. Failure to do so produces the growth rate $K=2$ regardless of whether the dynamics is regular or chaotic. The inclusion of the rotation $\theta$ kills off the linear growth.

In previous work [9] we illustrated the $0-1$ test for chaos with numerically obtained data sets by simulating maps, ordinary differential equations and partial differential equations, thereby showing the applicability to high-dimensional systems. Furthermore, we showed that a modified version of the test works very favorably for noisy systems when compared to traditional phase space reconstruction methods [10]. Here, we demonstrate the effectiveness of the modified test for experimental data.

To obtain experimental data, we constructed a bipolar motor, where a dipole magnet is suspended in a spatially uniform, linearly polarized oscillating magnetic field [3]. The motor consists of a short cylindrical ferrite permanent magnet located between a pair of Helmholtz coils so that it is free to rotate about an axis normal to the magnetic field of the coils, with the magnetic axis of the magnet perpendicular to the axis of rotation (see Fig. 1). The magnetic moment of this magnet was determined to be 3.70 joule/tesla by measuring the oscillation of the magnet in a fixed magnetic field. A magnetic field varying sinusoidally with frequency $\omega$ and peak value $4.91 \times$ $10^{-3}$ tesla was generated by driving the Helmholtz coils with a Pasco PI-9587C lowfrequency digital function generator/amplifier combination. We use the frequency $\omega$ 
as a parameter. To measure the angular position $\vartheta$ of the dipole magnet, we digitally recorded with a 25 frames/second digital video camera a spot painted on a aluminium disc mounted coaxially at the top of the rotor axle.

This experiment is the realization of the forced ordinary differential equation $\ddot{\vartheta}+$ $\gamma \dot{\vartheta}+(\mu B / I) \sin \vartheta \cos \omega t=0$, where $\vartheta$ is the angle between the dipole moment $\mu$ and the magnetic field $B$, and $I$ is the moment of inertia. Here, the damping is assumed to be proportional to the angular velocity with a constant rate $\gamma$. The observable $\Phi$ in the experiment corresponds to the function $\cos \vartheta$ (up to a constant phase).

Using $\Phi=\cos \vartheta$ as our observable, we took recordings for 9 minutes which amounts to 13500 data points. We measured the autocorrelation of the data and found them to be oversampled; the $e$-folding time [11] was $\tau=5$ and we therefore created a (shorter) time series consisting of 2700 data points. (We note that when the data is collected over a small interval of time, it is important that the data is not oversampled, since the mean square displacement scales as $M(n)=V n+o(n)$ where $V=0$ in the regular case and $V \ll 1$ in the oversampled chaotic case.) We found that our test works also with a data set recorded over 3 minutes, which amounts to $N=900$ data points after taking every 5 th data point.

In Fig. 2, we show experimentally obtained phase plots exhibiting periodic and chaotic dynamics for different values of the frequency $\omega$. One may question the need for our test as one can clearly distinguish chaotic from regular dynamics by looking at the phase plots in Fig. 2. However, this is possible only for low-dimensional systems, and even then it is problematic for systems with quasiperiodic dynamics. We showed previously [10] that our test distinguishes between quasiperiodic and chaotic dynamics in high dimensional systems. In this paper, it is our aim to show that the test works for real world problems which are inevitably contaminated by noise.

To illustrate the mathematics behind the test, we show in Fig. 3 the bounded/Brownianlike dynamics of the translation components $(p, q)$ of the $E(2)$ extension $(2)$ corresponding to the periodic/chaotic dynamics in Fig. 2. We made the arbitrary choice $c=1.95$ in computing the trajectories shown in Fig. 3. To produce this figure we took the data set consisting of 2700 points.

In Fig. 4, we show the mean square displacement corresponding to Fig. 3 (again for $c=1.95$ but now using the data set consisting of $N=900$ points) clearly showing the bounded behaviour for regular dynamics and the linear growth for chaotic dynamics. In practice [10], we take $n=10,11,12, \ldots, 90$ to ensure that $0 \ll n \ll N=900$ in the definition of $M(n)$. The asymptotic growth rate $K$ of the mean square displacement is determined by fitting a straight line to the curve $\log M(n)$ versus $\log n$ through minimizing the absolute deviation. This has the advantage compared to a least square fit that outliers are weighted less [15] which is desirable as the linear behaviour of $M(n)$ is only valid for $n \gg 1$ and can deviate strongly for small $n$.

Since the test is $2 \pi$-periodic in $c$, we choose $c$ from $(0,2 \pi)$. In Fig. 5 , we plot $K$ 
as a function of $c$ where $c$ is sampled uniformly with $\Delta c=0.005$ between 0.005 and 6.28. The figure shows that the test is essentially independent of the specific choice of $c$ and that we are justified in taking randomly chosen values of $c$. Only exceptional resonant values of $c$ yield values of $K$ which do not fit the picture of $K=0$ for regular dynamics and $K=1$ for chaotic dynamics. Resonances with a corresponding value of $K=2$ occur if the frequency $c$ is commensurate with a nonlinear frequency of the underlying dynamical system (in particular for values $c=0$ and $c=2 \pi$ for data with non-zero mean). Fig. 5 illustrates this resonance phenomenon. To avoid that these resonances distort the "true" value of $K$, we perform the test for several different values of $c$ and then take the median value of $K$. We use the median rather than the mean, since the median gives less weight to the strong outliers stemming from resonances. In practice, 100 random choices of $c$ suffices [10].

Using the experimental data and the method described above, we computed $K=$ 0.02 for the case $\omega=0.9 \mathrm{~Hz}$ and $K=0.92$ for the case $\omega=0.6 \mathrm{~Hz}$, clearly indicating regular dynamics for the first parameter value and chaotic dynamics for the second parameter value.

In Fig. 6, we show the dependence of the median value for $K$ as a function of $N$ for both cases. One can see clearly the improvement of the test with increasing $N$. Note that for the regular case in Fig. 6a, $K$ is not converging to 0 . This is due to the noise present in our data. However, values of 0.02 are sufficiently close to 0 to allow for a binary distinction with chaotic values around of $K$ which exceed 0.9. (See [10] for a discussion on data contaminated by measurement noise for our test.)

We have established a simple, inexpensive test for chaos and tested its efficiency with an analysis of experimental data obtained from a bipolar motor. The computational effort is of low cost, both in terms of programming efforts and in terms of actual computation time. This is a binary test that distinguishes purely between nonchaotic and chaotic dynamics by means of the quantity $K$ taking values close either to 0 or 1 . The most powerful aspects of our method are that there are no practical limitations arising from the nature and dimension of the deterministic data under consideration (except the intrinsic problem, mentioned earlier, that the time series needs to be sufficiently long that the dynamics explores enough of the attractor) and that the test is robust to contamination by noise.

Acknowledgments GAG is partly supported by ARC grant DP0452147. GAG and IM are partly supported by EPSRC grant GR/S22714/01. The authors are grateful for discussions with Ramon Xulvi-Brunet. 


\section{References}

[1] H.D.I. Abarbanel, R. Brown, J.J. Sidorovich \& L.S. Tsimring. The analysis of observed chaotic data in physical systems. Rev. Mod. Phys. 65 (1993) 1331-1392.

[2] P. Ashwin, I. Melbourne \& M. Nicol. Hypermeander of spirals; local bifurcations and statistical properties. Physica D 156 (2001) 364-382.

[3] M.J. Ballico, M.L. Sawley \& F. Skiff. The bipolar motor: A simple demonstration of deterministic chaos. Am. J. Phys. 58 (1990) 58-61.

[4] V.N Biktashev \& A.V. Holden. Deterministic Brownian motion in the hypermeander of spiral waves. Physica D 116 (2001) 342-382.

[5] H. Bruin, M. Holland \& I. Melbourne. Subexponential decay of correlations for compact group extensions of nonuniformly expanding systems. Ergod. Th. \& Dynam. Sys. 25 (2005) 1719-1738.

[6] M. Casdagli, S. Eubank, J.D. Farmer \& J. Gibson. State space reconstruction in the presence of noise. Physica D 51 (1991) 52-98.

[7] J.-P. Eckmann, S.O. Kamphurst, D. Ruelle \& S. Ciliberto. Liapunov exponents from time series. Phys. Rev. A 34 (1986) 4971-4979.

[8] M. Field, I. Melbourne \& A. Török. Decay of correlations, central limit theorems and approximation by Brownian motion for compact Lie group extensions. Ergod. Th. \& Dynam. Sys. 23 (2003) 87-110.

[9] G.A. Gottwald \& I. Melbourne. A new test for chaos in deterministic systems. Proc. Roy. Soc. A 460 (2004) 603-611.

[10] G.A. Gottwald \& I. Melbourne. Testing for chaos in deterministic systems with noise. Physica D 212 (2005) 100-110.

[11] H. Kantz and T. Schreiber. Nonlinear time series analysis. Cambridge University Press, 2nd edition, 2004.

[12] I. Melbourne \& M. Nicol. Statistical properties of endomorphisms and compact group extensions. Proc. London Math. Soc. 70 (2004) 427-446.

[13] M. Nicol, I. Melbourne \& P. Ashwin. Euclidean extensions of dynamical systems. Nonlinearity 14 (2001) 275-300.

[14] T.S. Parker \& L.O. Chua. Practical Numerical Algorithms for Chaotic Systems. Springer, New York, 1989. 
[15] W.H. Press, S.A. Teukolsky, W.T. Vetterling \& B.P. Flannery, Numerical Recipes in C. Cambridge University Press, 1992.

[16] M. Sano \& Y. Sawada. Measurement of the Lyapunov spectrum from a chaotic time series. Phys. Rev. Lett. 55 (1985) 1082-1085.

[17] T. Schreiber \& H. Kantz. Noise in chaotic data: diagnosis and treatment. Chaos 5 (1995) 133-142.

[18] F. Takens. Detecting strange attractors in turbulence. Lecture Notes in Mathematics 898, 366-381, Springer, Berlin, 1981.

[19] A. Wolf, J.B. Swift, H.L. Swinney \& J.A. Vastano. Determining Lyapunov exponents from a time series. Physica D 16 (1985) 285-317. 

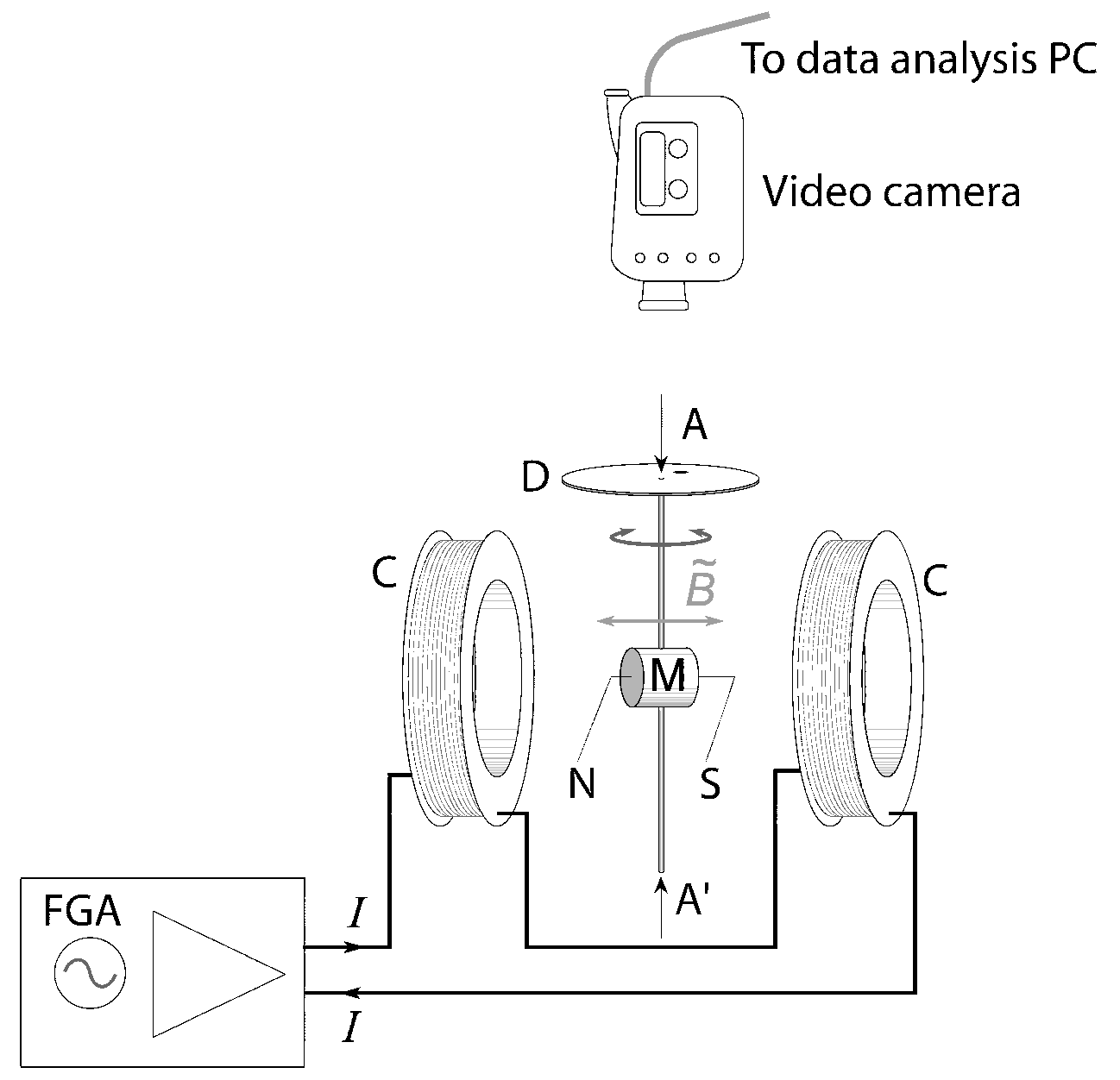

Figure 1: Schematic diagram of the experimental setup of the bipolar motor. C Helmholtz coil pair used to provide an oscillating magnetic field B. (For clarity, the coils are shown further apart than they would be in the Helmholtz configuration, where the (mean) coil separation is set equal to the (mean) coil radius.) FGA - Pasco PI-9587C function generator/amplifier; $\mathrm{M}$ - ferrite permanent magnet, where $\mathrm{N}$ and $\mathrm{S}$ indicate the orientation of its magnetic moment; D - indicator disc for angular position of the ferrite magnet. The position of the black spot on this white-painted disk is recorded by the video camera; $\mathrm{AA}^{\prime}$ - axis of rotation of the ferrite magnet. 

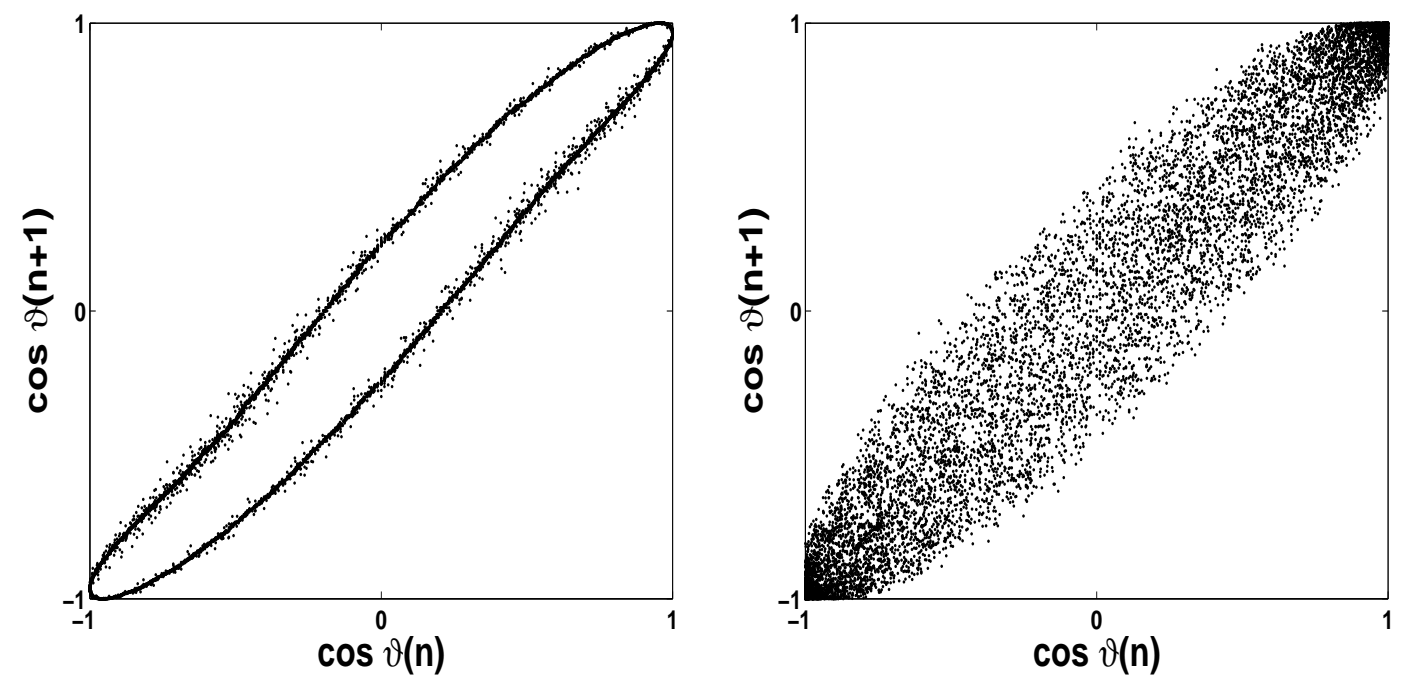

Figure 2: Experimentally obtained phase plots consisting of 13500 data points for different values of the forcing frequency $\omega$. The moment of inertia is $I=4.42 \times$ $10^{-4} \mathrm{~kg} \mathrm{~m}^{2}$, the magnetic moment is $\mu=3.70 \mathrm{~J} / \mathrm{T}$, the maximal magnetic field is $B=4.91 \times 10^{-3} \mathrm{~T}$ and the damping coefficient is $\gamma=0.046 \mathrm{~Hz}$. The forcing frequencies are (a) $\omega=0.9 \mathrm{~Hz}$, (b) $\omega=0.6 \mathrm{~Hz}$.
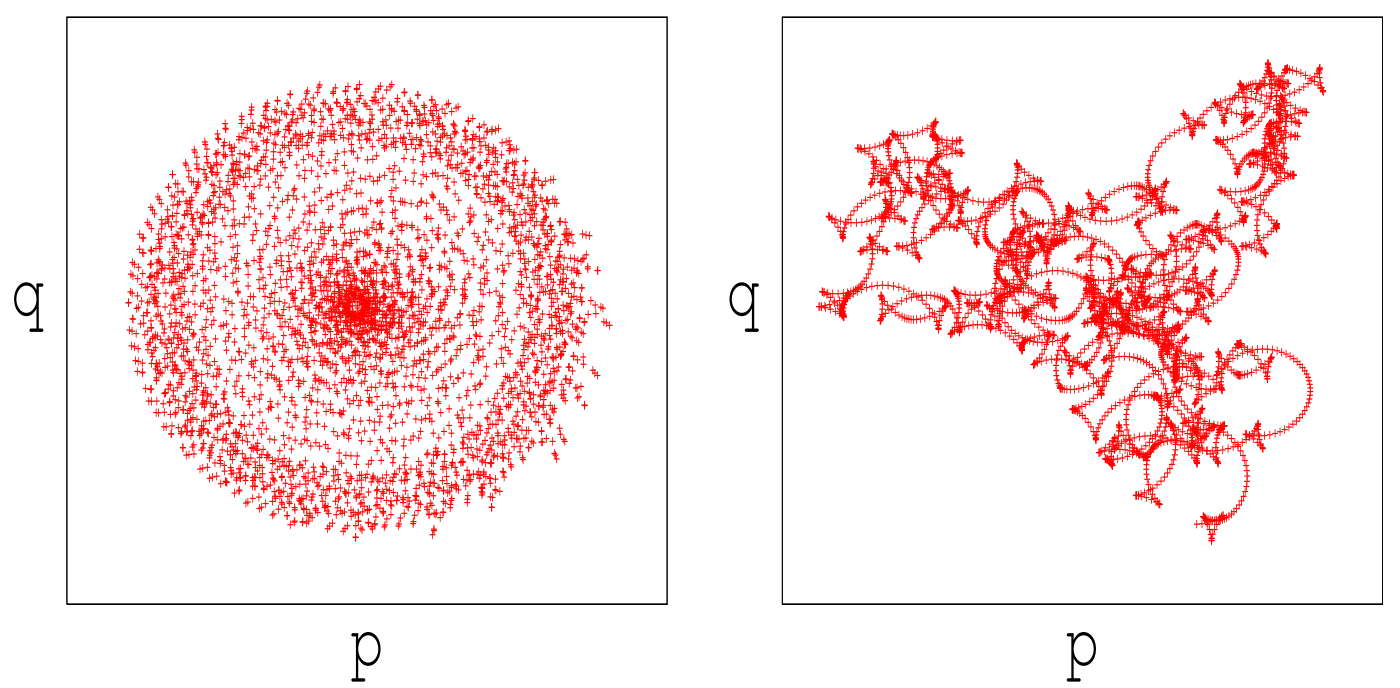

Figure 3: The dynamics of the translation components $(p, q)$ of the $E(2)$ extension. (a) bounded trajectories are shown corresponding to periodic dynamics at $\omega=0.9 \mathrm{~Hz}$. (b) Brownian-like trajectories are shown corresponding to chaotic dynamics at $\omega=0.6 \mathrm{~Hz}$. Both cases show 2700 data points and are calculated for $c=1.95$. 

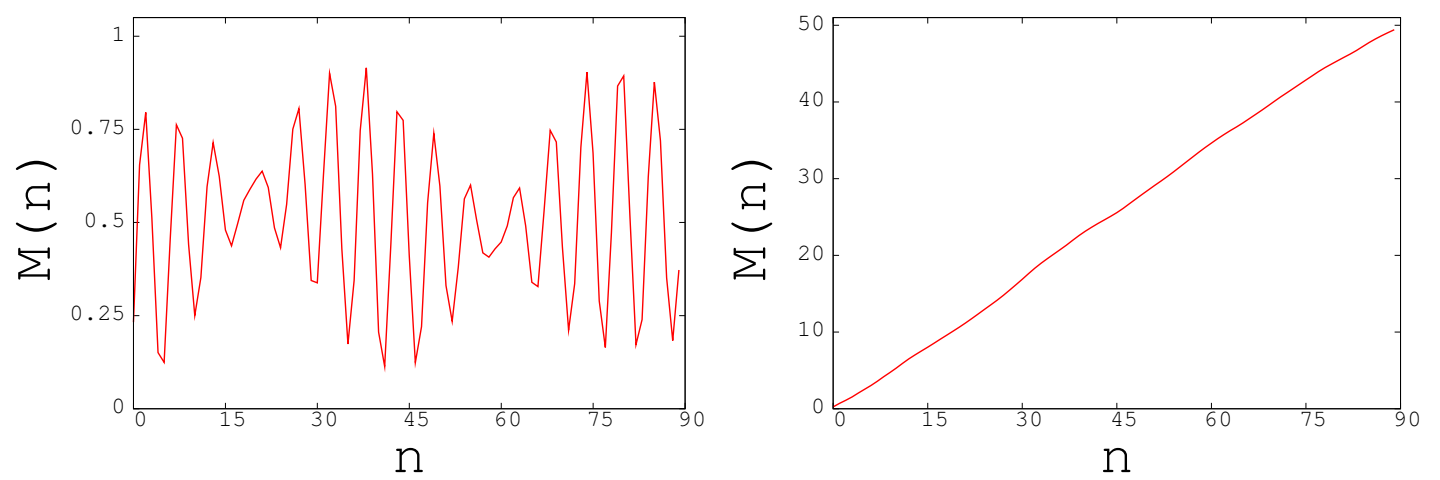

Figure 4: Mean square displacement $M(n)$ as a function of $n$ for 900 data points shown from $n=0$ up to $n=90$. (a) periodic case $\omega=0.9 \mathrm{~Hz}$ corresponding to Fig. 3a. (b) chaotic case $\omega=0.6 \mathrm{~Hz}$ corresponding to Fig. 3b. Both cases are calculated for $c=1.95$.
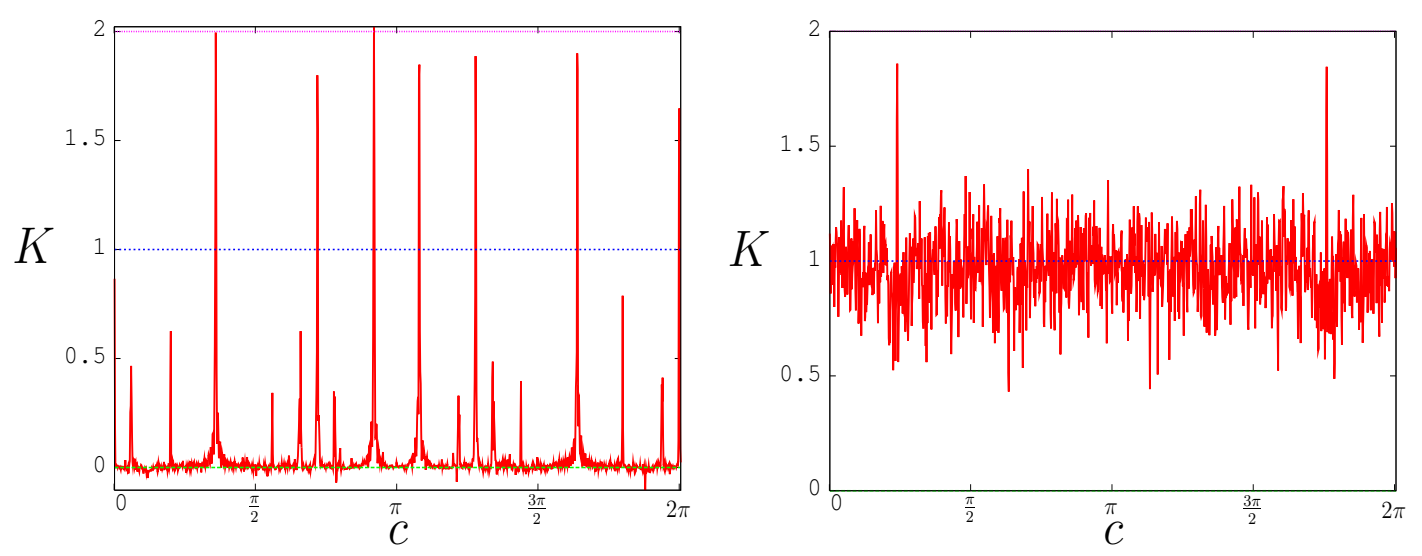

Figure 5: Dependence of the asymptotic growth rate $K$ on the frequency $c$. (a) regular dynamics at $0.9 \mathrm{~Hz}$ and (b) chaotic dynamics at $0.6 \mathrm{~Hz}$. 

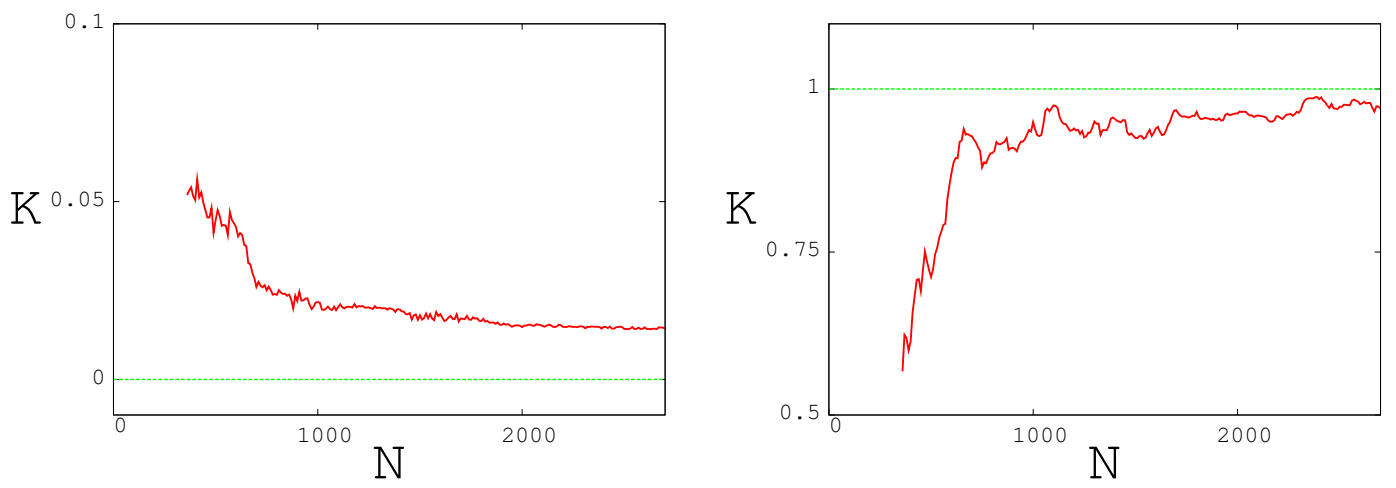

Figure 6: Dependence of the asymptotic growth rate $K$ on the length of the time series $N$. (a) periodic case $\omega=0.9 \mathrm{~Hz}$. (b) chaotic case $\omega=0.6 \mathrm{~Hz}$. We used the data set corresponding to the full 9 minutes of recording, taking every 5th data point, so the maximum value of $N$ is 2700 . 